\title{
A Two-Step MoM Procedure for the Analysis of Finite-Sized Tapered Slot Antennas
}

\author{
Arzu Keskin' Adnan Köksal \\ Department of Electrical and Electronics Engineering, Hacettepe University \\ 06800 Beytepe, Ankara, Turkey \\ arzukkeskin@gmail.com; koksal@ee.hacettepe.edu.tr
}

\begin{abstract}
An analysis of the Linearly Tapered Slot Antenna (LTSA) on a finite dielectric substrate has been carried out using a two step Method of Moments (MoM) procedure. In the first step of the analysis, the closed form Green's functions are obtained for the infinite dielectric substrate using the discrete complex image method. Galerkin type Method of Moments and RWG basis functions are used for the solution of the EFIE. In the second step, equivalent volume polarization currents in the substrate are used to modify the voltage vector for modeling the finite dielectric substrate region. The feed section of the antenna is modeled by a pulse excitation. Solving for the antenna currents with the modified excitation second time in air yields accurate results. Comparisons of radiation patterns for LTSAs on a finite dielectric substrate with HFFS results are presented and also a parametric study with respect to dimensions and permittivity are given.
\end{abstract}

Keywords: Linearly tapered slot antennas, integral equations, method of moments, closed form Green's function.

\section{Introduction}

The Linearly Tapered Slot Antenna which is suitable for microwave and millimeter wave system operates over a large bandwidth. Initial studies on the Linearly Tapered Slot Antenna were mostly experimental. Janaswamy who first presented the theoretical formulation for the analysis of the LTSA assumed the LTSA as infinite [1]. The actual geometry of the LTSAs on a finite dielectric substrate is analyzed by Köksal and Kauffman [2] using a full-wave MoM. In this work, a two-step MoM procedure is used for the analysis of LTSAs on a finite dielectric substrate.

Earlier studies on LTSA have shown that the patterns of LTSA's can be improved by changing the physical dimensions and other parameters of the antenna [3]. Therefore a parametric study is helpful. To validate the procedure given here, far field radiation patterns of the LTSA's are compared with the results of HFSS simulation and those reported in the literature.

\section{The Method}

This work is focused on obtaining the radiated far-fields of a single LTSA and investigating the effects of antenna parameters on the radiation pattern. The geometry of the LTSA on a finite-sized dielectric substrate is shown in Fig 1 . The ranges of antenna parameters are chosen as:

$$
\begin{gathered}
0.25 \lambda_{0} \leq L \leq 5 \lambda_{0} \\
0.25 \lambda_{0} \leq H \leq 3 \lambda_{0} \\
2.5^{0} \leq \alpha \leq 9^{0} \\
0.01 \lambda_{0} \leq d \leq 0.1 \lambda_{0} \\
1 \leq \varepsilon_{r} \leq 10.5
\end{gathered}
$$

The EFIE derived for the LTSA can be expressed as

$$
\begin{gathered}
\vec{E}_{\text {tan }}^{i}(\vec{r})=j \omega \int_{S} \overline{\bar{G}}^{A}\left(\vec{r}, \vec{r}^{\prime}\right) \cdot \vec{J}_{s}\left(\vec{r}^{\prime}\right) d s^{\prime}- \\
\quad 1 \\
\quad j \omega \int_{S} \nabla\left(\nabla \cdot \vec{J}_{s}\left(\vec{r}^{\prime}\right)\right) \cdot G^{q}\left(\vec{r}, \vec{r}^{\prime}\right) d s^{\prime}
\end{gathered}
$$

where $\vec{J}_{s}\left(\vec{r}^{\prime}\right)$ is the induced current density, $\vec{E}_{\text {tan }}^{i}$ is the tangential incident electric field, $\overline{\bar{G}}^{A}$ is the vector potential Green's function, $G^{q}$ is the scalar potential Green's function. Closed form spatial domain Green's functions are obtained using Sommerfeld identity shown in Eqn. (2) and 3-level DCIM [4],[5]. 


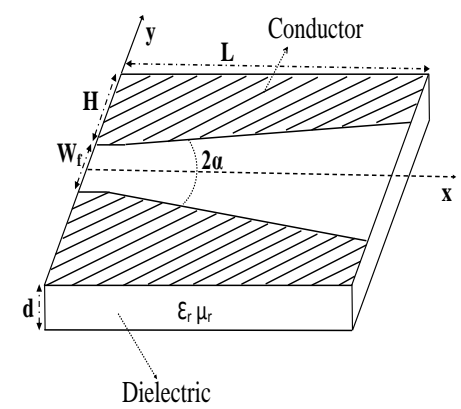

Fig. 1: LTSA Geometry

$$
\frac{e^{-j k r}}{r}=-\frac{j}{2} \int_{S I Y} d k_{\rho} k_{\rho} H_{0}^{(2)}\left(k_{\rho}\right) \frac{e^{-j k_{z}|z|}}{k_{z}}
$$

The closed-form approximations of spatial domain Green's functions are used in the EFIE given in Eqn. (1). LTSA conductor surface is modeled with a triangular mesh. RWG basis functions and Galerkin type MoM is used for the solution of the EFIE. The elements of the moment method matrix can be written using the Green's functions as

$$
\begin{aligned}
Z_{m n} & =j \omega \int_{S_{m}} f_{m}(\vec{r}) \cdot \int_{S_{n}} \overline{\bar{G}}^{A}\left(\vec{r}, \vec{r}^{\prime}\right) \cdot f_{n}\left(\vec{r}^{\prime}\right) d s^{\prime} d s \\
& -\frac{1}{j \omega} \int_{S_{m}} \nabla \cdot f_{m}(\vec{r}) \int_{S_{n}} G^{q}\left(\vec{r}, \vec{r}^{\prime}\right) \nabla^{\prime} \cdot f_{n}\left(\vec{r}^{\prime}\right) d s^{\prime} d s
\end{aligned}
$$

The unknown conductor currents are the solution of the MoM matrix equation. In order to account for the finiteness of the substrate the equivalent polarization currents are found as:

$$
\vec{J}_{p}=j \omega\left(\varepsilon-\varepsilon_{0}\right) \vec{E}
$$

Cubical segmentation is utilized for the dielectric part of the antenna, and the polarization current is expanded with pulses. Hence the $\vec{E}$ field in (4) is evaluated in the middle of each cubical section of the dielectric substrate to obtain the values of the pulses.

In the second step of the procedure, the effect of the polarization currents on the voltage vector is calculated. The excitation vector is modified by the amount given as:

$$
\Delta V_{m}=\int_{S c} \vec{E}\left\{\vec{J}_{p}\right\} \cdot \vec{J}_{c} d s
$$

The EFIE is solved in air with the new incident field vector this time to obtain the final surface current density on the LTSA. By using the outlined procedure, the radiation characteristics of the LTSAs have been analyzed.

\section{Results and Discussion}

The method presented in the previous section is cast into a MoM code. The validity of the model is demonstrated by obtaining the $\mathrm{E}$ and $\mathrm{H}$ plane patterns of LTSA and by comparing our results to those of simulation program HFSS. in Fig. 1.

E-plane of the LTSA coincides with the $x-y$ plane, and the H-plane is the $x-z$ plane for the coordinate system shown 


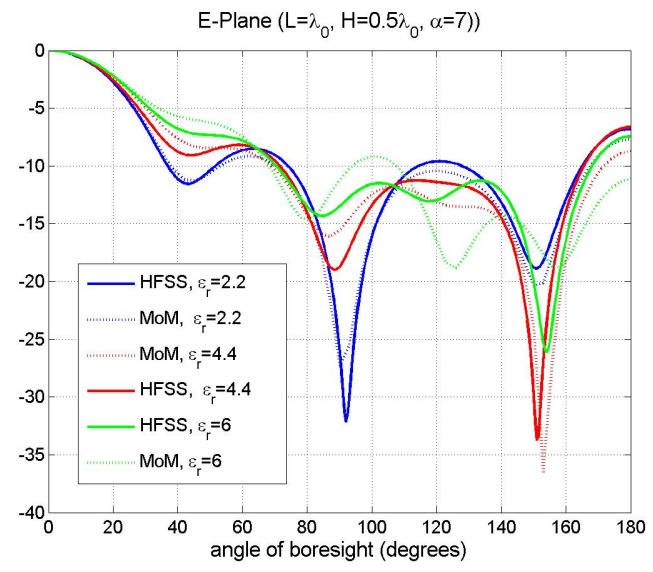

Fig. 2: Variation of the E-Plane pattern for an LTSA with $\varepsilon_{r}=2.2, \varepsilon_{r}=4.4, \varepsilon_{r}=6\left(L=\lambda_{o}, H=0.5 \lambda_{o}, W_{f}=0.012 \lambda_{o}, d=0.017 \lambda_{o}, \alpha=\right.$ $\left.7^{\circ}\right)$.

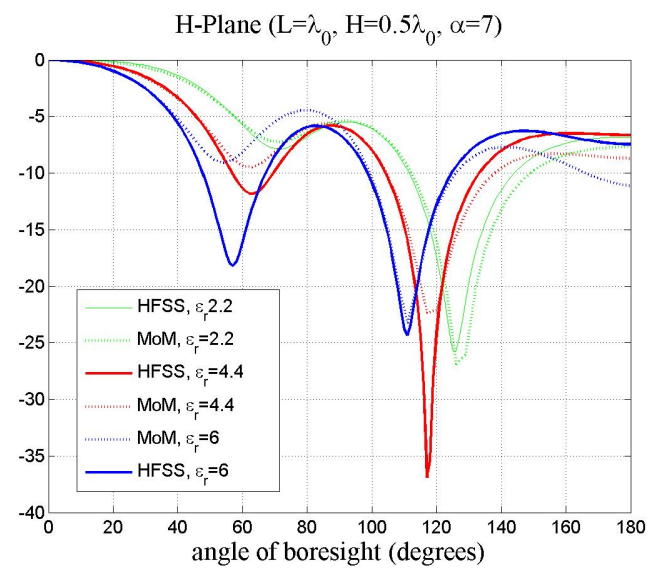

Fig. 3: Variation of the H-Plane pattern for an LTSA with $\varepsilon_{r}=2.2, \varepsilon_{r}=4.4, \varepsilon_{r}=6\left(L=\lambda_{o}, H=0.5 \lambda_{o}, W_{f}=0.012 \lambda_{o}, d=0.017 \lambda_{o}, \alpha=\right.$ $\left.7^{\circ}\right)$.

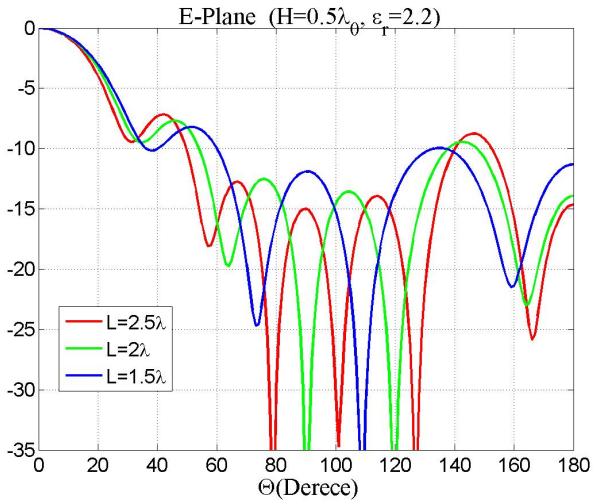

Fig. 4: Radiation patterns for an LTSA with $L=1.5 \lambda_{o}, L=2 \lambda_{o}, L=2.5 \lambda_{o},\left(H=0.5 \lambda_{o}, W_{f}^{(b)}=0.012 \lambda_{o}, d=0.017 \lambda_{o}, \varepsilon_{r}=2.2, \alpha=7^{o}\right)$.

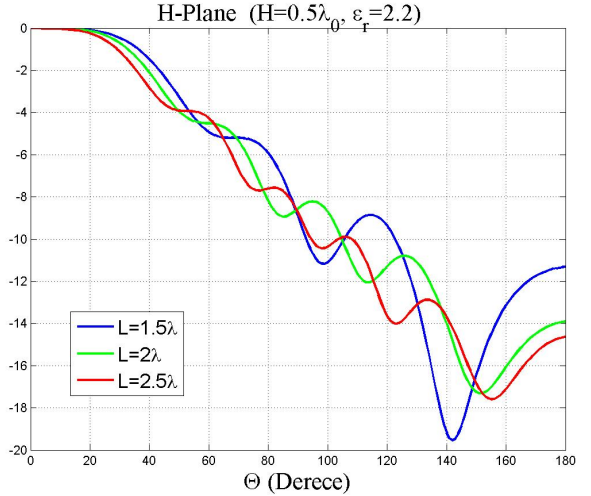

Fig. 4: Radiation patterns for an LTSA with $L=1.5 \lambda_{o}, L=2 \lambda_{o}, L=2.5 \lambda_{o},\left(H=0.5 \lambda_{o}, W_{f}^{(b)}=0.012 \lambda_{o}, d=0.017 \lambda_{o}, \varepsilon_{r}=2.2, \alpha=7^{o}\right)$.

To see the effect of the antenna parameters on the radiation pattern, a parametric study of the LTSA is carried out. Firstly, the effect of the dielectric constant has been analyzed as shown in Figs. 2 and 3. These figures show that as the per- 


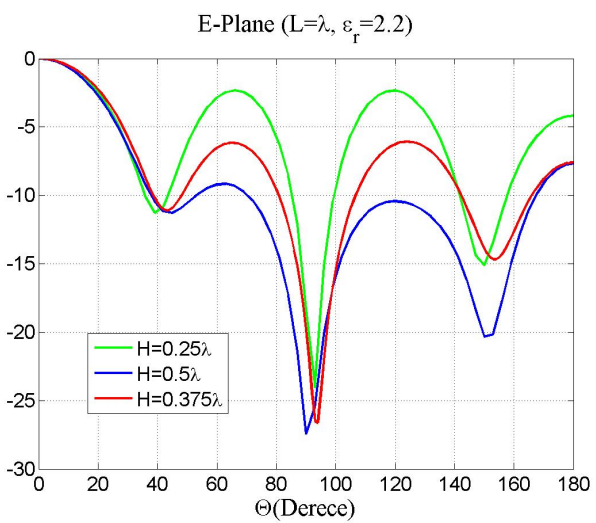

(a)

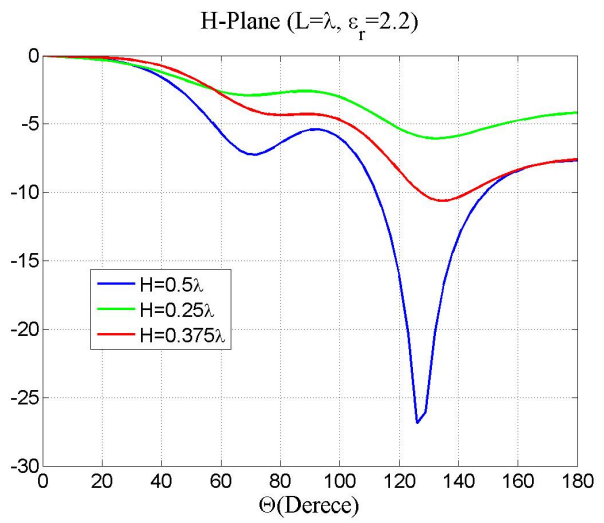

(b)

Fig. 5: Radiation patterns for an LTSA with $H=0.25 \lambda_{o}, H=0.375 \lambda_{o}, H=0.5 \lambda_{o},\left(L=\lambda_{o}, W_{f}=0.012 \lambda_{o}, d=0.017 \lambda_{o}, \varepsilon_{r}=2.2, \alpha=7^{o}\right)$.

mittivity increases, sidelobe level and the beamwidth in the E-Plane increases, while the beamwidth in the H-Plane decreases. Secondly, the effect of antenna dimensions are given in Figs. ?? and ?? which show that the side lobe level in the E-plane increases as the length of the LTSA increases while the beamwidth in H-plane decreases. Similarly, the side lobe level in the E-plane decreases in E and H plane as the width of the LTSA increases as shown in Figs. ?? and ??

\section{Conclusion}

In this work, a two-step MoM procedure is presented for LTSAs on a finite dielectric substrate. In this method the radiation characteristic of the antenna is analyzed by using a two step model. In the first step the conductor antenna currents are obtained by solving the electric field integral equation. The dielectric substrate is assumed as infinite and Method of Moments is used for the solution EFIE. In the second step, the effect of the finite dielectric region is modelled with volume polarization currents and using these polarization currents the excitation vector is modified. Then the conductor currents with this new voltage vector are obtained in the air.

The results obtained using the presented method display good agreement with HFSS results.

It is worthwhile to mention that, the method reported in this paper can be applied directly to other antennas with finite-sized dielectric substrate, for example to microstrip patch antennas.

\section{References}

[1] R. Janaswamy, "An Accurate Moment Method Model for The Tapered Slot Antenna, IEEE Trans. Antennas Propagat., vol. AP-37, pp. 1523-1528, 1989.

[2] A. Köksal and J. F. Kauffman, "Moment Method Analysis of Linearly Tapered Slot Antennas," Int Journal of Microwave and Millimeter-Wave CAE 4, pp. 76-87, 1994.

[3] A. Köksal, "Characterization and Optimization of Linearly Tapered Slot Antenna Pattern," Int Journal of Infrared and Millimeter-Wave CAE 4, vol. 18, no. 8, pp. 1525-1537, 1997.

[4] M. I. Aksun and N. Kinayman, "Modern Microwave Circuits," Artech House, 2005.

[5] A. Alparslan, Study of Green's Functions of Potentials and Fields in Layered Media Composed of Left-handed and Right-handed Materials, M.Sc Thesis, Koc̣ Üniversitesi, Turkey, 2008. 